\title{
Ecological Adaptation Moderates the Temperature-Sensitivity of the Biological Carbon Pump
}

\author{
Daniel E. GASKELL ${ }^{1 *}$, MOJTABA FAKHRAEE ${ }^{2}$, NOAH \\ PLANAVSKY ${ }^{2}$, PINCELLI M. HULL ${ }^{2}$ \\ ${ }^{1}$ Yale University, New Haven, CT (*correspondence: \\ daniel.gaskell@yale.edu) \\ ${ }^{2}$ Yale University, New Haven, CT
}

A large body of evidence supports the hypothesis that organic carbon degradation is temperature-dependent: as temperatures rise, so do the metabolic rates of remineralizers, resulting in a shallower average depth of remineralization and reduced organic carbon export to the deep ocean. This creates a positive climate feedback which must be considered when modeling the carbon cycle of warmer, high- $\mathrm{CO}_{2}$ worlds. However, the magnitude of this effect remains unclear, as the temperature-sensitivity of microbial metabolisms may change as organisms adapt to a hotter world. Insight can be gained from the carbon export regime of past high- $\mathrm{CO}_{2}$ worlds. We apply a novel multi-pronged approach for constraining the temperature-sensitivity of remineralization by combining $\delta^{13} C_{\text {DIC }}$ proxy data from Eocene planktonic foraminifera with mechanistic models of both organic carbon remineralization and the biochemistry of the foraminifera used to construct the proxy record. We present evidence that higher temperatures do reduce organic carbon export, but that this effect is moderated by apparent temperature adaptation within the microbial community. 